San Jose State University

SJSU ScholarWorks

Faculty Publications

Electrical Engineering

1995

\title{
Multilevel Block Coded 8-PSK Modulations Using Unequal Error Protection Codes for the Rayleigh Fading Channel
}

\author{
Robert H. Morelos-Zaragoza \\ University of Tokyo, robert.morelos-zaragoza@sjsu.edu \\ Tadeo Kamasi \\ Nara Institute of Science and Technology \\ Shu Lin \\ University of Hawaii at Manoa
}

Follow this and additional works at: https://scholarworks.sjsu.edu/ee_pub

Part of the Electrical and Computer Engineering Commons

\begin{abstract}
Recommended Citation
Robert H. Morelos-Zaragoza, Tadeo Kamasi, and Shu Lin. "Multilevel Block Coded 8-PSK Modulations Using Unequal Error Protection Codes for the Rayleigh Fading Channel" Faculty Publications (1995): 486-490. https://doi.org/10.1109/PIMRC.1995.480916
\end{abstract}

This Article is brought to you for free and open access by the Electrical Engineering at SJSU ScholarWorks. It has been accepted for inclusion in Faculty Publications by an authorized administrator of SJSU ScholarWorks. For more information, please contact scholarworks@sjsu.edu. 


\title{
Multilevel Block Coded 8-PSK Modulations Using Unequal Error Protection Codes for the Rayleigh Fading Channel
}

\author{
Robert Morelos-Zaragoza ${ }^{\star}$ \\ Tadao Kasami ${ }^{\dagger}$ \\ Shu $\operatorname{Lin}^{\ddagger}$ \\ * Institute of Industrial Science, University of Tokyo, 7-22-1 Roppongi, Minatoku, Tokyo 106 Japan \\ $\dagger$ Graduate School of Info. Science, Nara Institute of Science and Tech., Ikoma, Nara 630-01 Japan \\ ‡ Department of Electrical Engineering, University of Hawaii, Honolulu, Hawaii 96822 U.S.A.
}

\begin{abstract}
This paper introduces new block coded 8-PSK modulations with unequal error protection (UEP) capabilities for Rayleigh fading channels. The design of efficient block coded modulations (BCM) over 8-PSK signal sets, it for the specific purpose of UEP, over Rayleigh fading channels is considered. UEP is desirable in communications systems where part of the source information is more important, or error sensitive, such as transmission of coded speech and data broadcasting. The proposed block modulation codes are based on the multilevel construction of Imai and Hirakawa [1]. It is shown that the use of binary linear UEP (LUEP) codes [2] as component codes in one or two of the encoding levels provides, in addition to superior UEP capabilities, a higher error performance, at the expense of a very modest reduction in bandwidth efficiency, with respect to conventional multilevel codes. Computer simulation results show that, over a Rayleigh fading channel, a significant improvement in coding gain is obtained by the use of binary LUEP codes as constituent codes in the multilevel construction.
\end{abstract}

\section{INTRODUCTION}

Previous work on combining LUEP codes and PSK modulation for fading channels is reported in references [3] and [4]. Hagenauer et al. [3] proposed ratecompatible punctured convolutional codes combined with DQPSK modulation to provide UEP by means of their inherent variable rate structure. In a previous paper [4] we used Gray labeling of a QPSK signal set to map binary LUEP codes of even length onto block modulation codes with UEP capabilities. Seshadri and Sundberg [5] studied the UEP capabilities of the Imai-Hirakawa multilevel construction over Rayleigh fading channels with binary linear codes of length 8 and nonuniform Gray mapped 8-PSK signal sets. In [6] this study was extended to multilevel trellis coded modulation.

The aim of this research work is to design efficient block coded modulations (BCM) over 8-PSK signal sets for the specific purpose of UEP over Rayleigh fading channels. The proposed block modulation codes are compared with the best known multilevel BCM using conventional linear block codes [5][9] of the same length and same minimum product and symbol distances, over Rayleigh fading channels.

It is well known that over a fading channel, the minimum symbol and product distances are the parameters that dominate the overall error performance [7]. The product distance determines the error performance at low signal-to-noise ratios (SNR), while the symbol distance dominates at moderate to high SNR and is closely related to the Hamming distance of the component codes. Thus it is natural to use binary LUEP codes as component codes in the multilevel construction to obtain good BCM for UEP over fading channels. To illustrate the proposed modulation codes and their performance, two examples are presented. A detailed analysis of the effects of increasing the symbol and product distances in one or more stages of the multilevel construction is a difficult task for Rayleigh fading channels. In this paper, computer simulation results are reported to show that a significant improvement in coding gain is obtained by the use of an LUEP code as constituent code in the multilevel construction. In the computer simulations we assume a flat slow fading channel, with independent Rayleigh distributed fading amplitudes, and perfect frequency and phase synchronization.

\section{PRELIMINARIES}

Let $S$ represent a uniform unit-energy 8-PSK signal set (see Figure 1). In this paper, natural labeling (i.e., standard mapping by set partitioning) of set $S$ is considered. That is, a label $\ell_{k}=b_{1}+2 b_{2}+4 b_{3}$ represents the signal point $e^{j k \pi / 4}$, for $0 \leq k<8$, where $j=\sqrt{-1}$, and $b_{i} \in\{0,1\}, 1 \leq i \leq 3$. In multilevel 


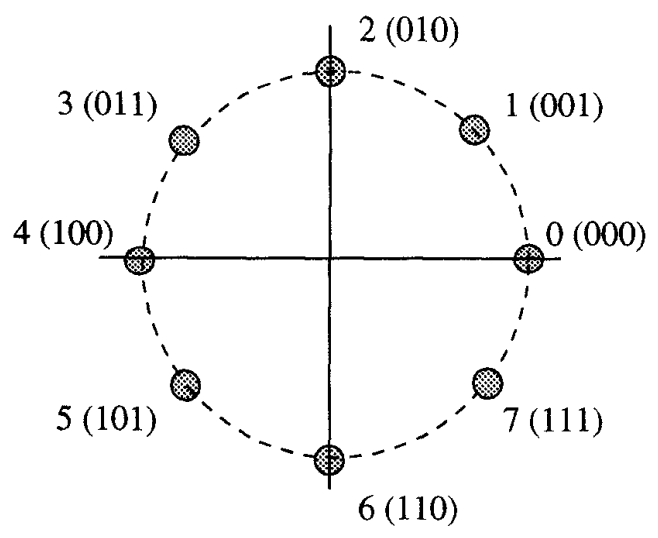

FIGURE 1: An 8-PSK signal constellation with natural labeling

block coded modulation [1], codewords of three linear codes of length $n$, dimension $k_{i}$ and minimum distance $d_{i}$, denoted $C_{i}$, are used to select label bits $b_{i}$, for $1 \leq i \leq 3$. The set of resulting sequences of 8-PSK signals is said to be a block modulation code $\boldsymbol{\Lambda}$ of length $n$ and rate (or bandwidth efficiency) $R=\left(k_{1}+k_{2}+k_{3}\right) / n$ bits/symbol.

Throughout the paper binary LUEP codes are used. For simplicity, only LUEP codes with two levels of error protection are considered. A two-level $(n, k)$ LUEP code is a linear code that it is not spanned by its set of minimum weight vectors [8]. We use $\operatorname{UEP}(n, k)$ to denote such a code and refer to its unequal error protection capabilities as follows: separation vector $\overline{\mathbf{s}}=\left(s_{1}, s_{2}\right)$ for the message space $\{0,1\}^{k^{(1)}} \times\{0,1\}^{k^{(2)}}$, where $k=k^{(1)}+k^{(2)}$. This is to say that codewords in correspondence to $k^{(i)}$ information bits are at a Hamming distance at least $s_{i}$, $i=1,2$. Without loss of generality, we assume that $s_{1} \geq s_{2}$. Then $k_{2}$ is equal to the dimension of the span of the minimum weight codewords of $\operatorname{UEP}(n, k)$. In other words, an information vector of length $k$ bits can be separated into a most significant part of length $k^{(1)}$ bits (the MSB) and a least significant part of length $k^{(2)}$ bits (the LSB).

Figure 2 shows the block diagram of an encoder for the proposed multilevel modulation codes. Conventional $\left(n, k_{1}, d_{1}\right)$ and $\left(n, k_{2}, d_{2}\right)$ linear block codes $C_{1}$ and $C_{2}$ are selected to ensure that the minimum

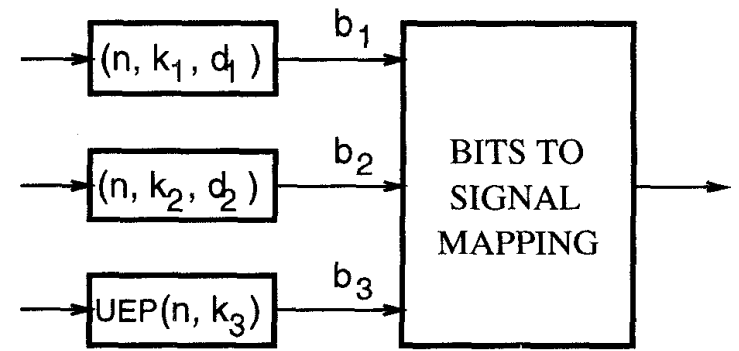

FIGURE 2: An encoder for the proposed multilevel modulation codes for UEP

symbol distarce

$$
\delta_{H} \triangleq \min \left\{d_{1}, d_{2}, d_{3}\right\}
$$

will occur at the second or third encoding levels, so that the minimum product distance

$$
\Delta_{p}^{2} \triangleq\left(\delta_{k}\right)^{d_{k}}, k=\min \left\{i: \delta_{H}=d_{i}\right\}
$$

will be greater than 2 , where $\delta_{1}=0.586, \delta_{2}=2$ and $\delta_{3}=4$. Details on these and other design considerations can be found in [7] and [9]. The proposed multilevel construction uses a $\operatorname{UEP}\left(n, k_{3}\right)$ code $C_{3}$ in the third encoding level. In sections 3 and 4 , we present example block modulation codes of lengths 8 and 32 to illustrate the proposed construction and its error performance in comparison with other approaches.

In comparing the proposed 8-PSK block modulation codes with conventional ones, the decoding complexity is also used as a measure. For maximum likelihood soft-decision decoding (MLSD), the Viterbi algorithm may be used, operating on a trellis diagram $T$ for a modulation code $\boldsymbol{\Lambda}$. Much is now known on the structure of trellis diagrams for some classes of linear block codes, notably of Reed-Muller codes (see the list of references in paper [9]). In this paper, we measure the decoding complexity by the number of states of a minimal trellis diagram $T_{\min }$ of $\boldsymbol{\Lambda}$. For each encoding stage of the multilevel construction, let $2^{s_{i}}$ be the number of states of a minimal trellis diagram for code $C_{i}, i=1,2,3$. Then the decoding complexity of $\boldsymbol{\Lambda}$ (number of states of $\left.\mathrm{T}_{\min }\right)$ is $2^{s_{1}} \times 2^{s_{2}} \times 2^{s_{3}}=2^{s_{1}+s_{2}+s_{3}}$. It is also well known [5] that suboptimal multistage soft-decision decoding (MSDD) reduces significantly the decoding 
complexity at the expense of a very modest reduction in coding gain. With suboptimal MSDD, the decoding complexity of $\Lambda$ is given by $2^{s_{1}}+2^{s_{2}}+2^{s_{3}}$.

\section{A LENGHT-8 MULTILEVEL CODE FOR UEP OVER RAYLEIGH FADING CHANNELS}

Let $C_{1}, C_{2}$ and $C_{3}$ be $(8,4,4),(8,7,2)$ and $(8,7,2)$ linear codes, respectively. The Imai-Hirakawa multilevel construction results in a block modulation code $\boldsymbol{\Lambda}_{\mathbf{1}}$ of length 8 , rate $R=2.25 \mathrm{bits} / \mathrm{symbol}$, minimum symbol distance $\delta_{H}=2$ and minimum product distance $\Delta_{p}^{2}=4$. This code was analyzed in [5] and its UEP capabilities exhibited through computer simulations.

By letting $C_{3}$ be a binary optimal LUEP code, $\operatorname{UEP}(8,5)$, from $[10]$ with separation vector $\overline{\mathbf{s}}=(3,2)$ for the message space $\{0,1\}^{4} \times\{0,1\}$, a block modulation code $\boldsymbol{\Lambda}_{\mathbf{2}}$ is obtained. A (trellis oriented) generator matrix for $C_{3}$ is given by

$$
G_{3}=\left(\begin{array}{l}
10100101 \\
01100100 \\
00110010 \\
00001110 \\
00000011
\end{array}\right),
$$

and it can be easily verified that any two codewords of $C_{3}$, in correspondence to information vectors whose first 4 information bits (or, equivalently, the first 4 rows of $G_{3}$ ) differ, are at a Hamming distance of at least 3. Modulation code $\boldsymbol{\Lambda}_{2}$ has length 8 , rate $R=2 \mathrm{bits} / \mathrm{symbol}, \delta_{H}=2$ and $\Delta_{p}^{2}=4$. In addition, $25 \%$ of the information bits (the 4 MSB encoded by $\operatorname{UEP}(8,5))$ have corresponding symbol and product distances equal to 3 and 64 , respectively. That is, a subset of the coded sequences, those corresponding to the MSB encoded by the LUEP code, have higher symbol and product distances than the conventional code $\boldsymbol{\Lambda}_{\mathbf{1}}$. It follows that, with no bandwidth expansion over uncoded QPSK, higher error performance is achieved (See also the simulation results shown in Figure 3).

In terms of decoding complexity, it can be shown that MLSD of $\boldsymbol{\Lambda}_{1}$ requires a trellis diagram of $2^{2} \times 2 \times 2=2^{4}$ states, while that of $\boldsymbol{\Lambda}_{2}$ requires $2^{2} \times 2 \times 2^{2}=2^{5}$ states. It follows that the improvement in coding gain and UEP capabilities is attained roughly by doubling the decoding complexity. However, the use of suboptimal MSDD results in comparable decoding complexity for both codes: The number of states of a minimal trellis diagram for use in MSDD for $\boldsymbol{\Lambda}_{\mathbf{1}}$ and $\boldsymbol{\Lambda}_{\mathbf{2}}$ is $2^{2}+2+2=\mathbf{8}$ and $2^{2}+2+2^{2}=10$, respectively. Therefore with the use of suboptimal multistage decoding, the proposed modulation code of length 8 would require only $25 \%$ more decoding complexity than conventional BCM. It is worthwhile noting that in multistage decoding of both $\boldsymbol{\Lambda}_{\mathbf{1}}$ and $\boldsymbol{\Lambda}_{\mathbf{2}}$, because the first two codes, $(8,4,4)$ and $(8,7,2)$ linear codes, are identical, the same reduction of coding gain will be present in the first two decoding stages. The third stage of $\boldsymbol{\Lambda}_{\mathbf{2}}$ provides enhanced UEP capabilities.

The multilevel 8-PSK modulation code $\boldsymbol{\Lambda}_{\mathbf{2}}$ above is compared with a multilevel code for UEP using conventional linear codes with about the same overall error performance: Time-sharing of $(7,4,3)$ and $(2,1,2)$ linear codes, which produces a $\operatorname{UEP}(9,5)$ code, denoted $|(7,4,3)|(2,1,2) \mid$, is used as $C_{3} . \quad C_{1}$ and $C_{2}$ are $(9,4,4)$ and $(9,8,2)$ linear codes, respectively. A block modulation code $\boldsymbol{\Lambda}_{\mathbf{3}}$ of length 9 and rate $R=1.89 \mathrm{bits} /$ symbol results, with the same minimum symbol and product distances as $\boldsymbol{\Lambda}_{\mathbf{2}}$, but reduced bandwidth efficiency and slightly higher decoding complexity (a block length of 9 ).

Computer simulation results for $\boldsymbol{\Lambda}_{\mathbf{1}}$ (EEP) and $\boldsymbol{\Lambda}_{\mathbf{2}}$ (UEP) are shown in Figure 3. The results were obtained using a uniform 8-PSK signal set with natural labeling and single-stage maximum likelihood softdecision decoding using the Viterbi algorithm. The increase in coding gain for the most important message part is very impressive. At a bit error rate (BER) of $10^{-3}$, the coding gain in the third stage is at least $13 \mathrm{~dB}$ for $\boldsymbol{\Lambda}_{\mathbf{2}}$, compared to about $8.5 \mathrm{~dB}$ for $\boldsymbol{\Lambda}_{\mathbf{1}}$. Note that for the conventional multilevel code $\boldsymbol{\Lambda}_{1}$, the largest coding gain at a BER of $10^{-3}$ occurs in the 4 bits encoded by the first stage (Pe1). This coding gain is about $11.5 \mathrm{~dB}$ compared to $14 \mathrm{~dB}$ in the third encoding stage (the $4 \mathrm{MSB}$ encoded by the LUEP code) for $\mathbf{\Lambda}_{\mathbf{2}}$. In addition, the overall coding gain for the proposed construction at a BER of $10^{-3}$ is about $2 \mathrm{~dB}$ larger than for the conventional multilevel code.

\section{A LENGTH-32 MULTILEVEL CODE FOR UEP OVER RAYLEIGH FADING CHANNELS}

Let $C_{1}, C_{2}$ and $C_{3}$ be $(32,16,8),(32,26,4)$ and $(32,26,4)$ Reed-Muller (RM) codes, respectively. The multilevel construction yields a block modulation code $\Lambda_{4}$ of length 32 , rate $R=2.125$ bits/symbol, 


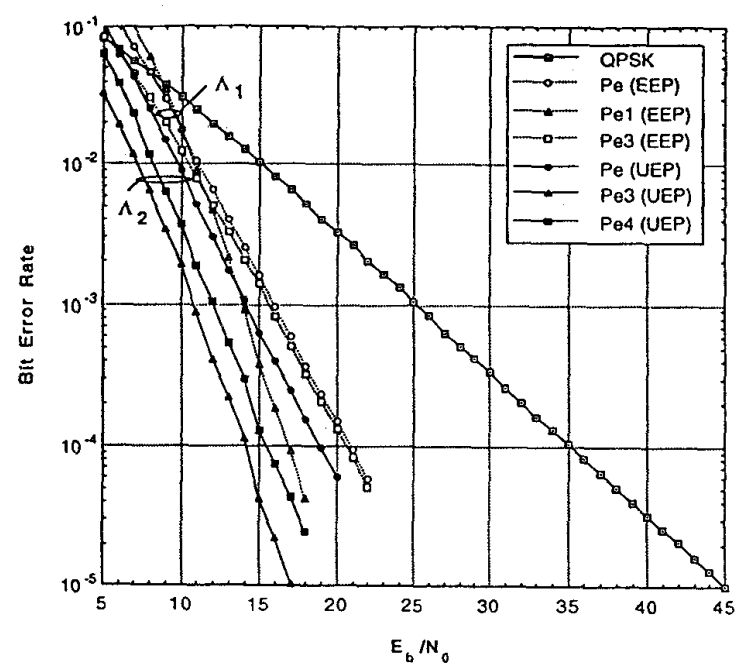

FIGURE 3: Simulation results for $\boldsymbol{\Lambda}_{\mathbf{1}}$ (EEP) and $\boldsymbol{\Lambda}_{\mathbf{2}}$ (UEP). (Pe: Overall bit error rate (BER), Pe1: BER in stage 1, Pe3: BER in stage 3 for the MSB, Pe4: BER in stage 3 for the LSB).

minimum symbol distance $\delta_{H}=4$ and minimum product distance $\Delta_{p}^{2}=16$. This code was analyzed in [9] and shown to achieve high performance over a Rayleigh fading channel.

Let $C_{1}$ and $C_{2}$ be as above and let $C_{3}$ be a $\operatorname{UEP}(32,22)$ code with separation vector $\overline{\mathrm{s}}=(6,4)$ for the message space $\{0,1\}^{7} \times\{0,1\}^{15}$. This $\operatorname{UEP}(32,22)$ code is obtained from combining an extended $(16,7,6) \mathrm{BCH}$ code and a $(16,15,2) \mathrm{RM}$ code using the $|u| u+v \mid$ construction [4]. The result is a block modulation code $\boldsymbol{\Lambda}_{\mathbf{5}}$ of length 32 , rate $R=2 \mathrm{bits} / \mathrm{symbol}, \delta_{H}=4$ and $\Delta_{p}^{2}=16$, that provides coded sequences in correspondence to $10.94 \%$ of the information (the $7 \mathrm{MSB}$ encoded by $C_{3}$ ) with symbol and product distances of 6 and 4096, respectively. This results in enhanced UEP capabilities and higher overall error performance with respect to $\mathbf{\Lambda}_{\mathbf{4}}$, with the same bandwidth efficiency as uncoded QPSK modulation.

$\boldsymbol{\Lambda}_{\mathbf{5}}$ is compared with BCM for UEP using conventional linear block codes as follows: To obtain the same error protection capabilities as the UEP code $C_{3}$ used by $\Lambda_{4}$, the time-sharing of $(16,7,6)$ and $(21,15,4)$ linear codes, resulting in a $\operatorname{UEP}(37,22)$ code, denoted $|(16,7,6)|(21,15,4) \mid$, may be used. Let $C_{1}$ be a $(37,22,8)$ linear code from the table of best linear codes [11], $C_{2}$ be a $(37,30,4)$ linear code (a shortened Hamming code), and $C_{3}$ be the $|(16,7,6)|(21,15,4) \mid$ code. The multilevel construction results in a modulation code $\mathbf{\Lambda}_{\mathbf{6}}$ of length 37 and rate $R=2$ bits/symbol, i.e., the same rate and error protection capabilities as $\boldsymbol{\Lambda}_{\mathbf{5}}$. However, $\boldsymbol{\Lambda}_{\mathbf{6}}$ has higher decoding complexity (a block length of 37 ) than that of $\mathbf{\Lambda}_{\mathbf{5}}$.

For MLSD using the Viterbi algorithm, the decoding complexity (as defined in Section 2) of $\boldsymbol{\Lambda}_{4}$ is $2^{9} \times 2^{4} \times 2^{4}=2^{17}$. For $\Lambda_{5}$ the binary LUEP code used in the third stage of the multilevel construction introduces additional decoding complexity. In the third stage, a minimal trellis diagram for the $\operatorname{UEP}(32,22)$ code requires $2^{7}$ states, as opposed to $2^{4}$ for the $(32,26,4) \mathrm{RM}$ code used in $\mathbf{\Lambda}_{4}$. The decoding complexity of the proposed BCM for UEP of length 32 is thus roughly eight times that of conventional BCM (The increased decoding complexity of the UEP code in the third stage). However, with the use of multistage decoding the decoding complexity of $\boldsymbol{\Lambda}_{4}$ is $2^{9}+2^{4}+2^{4}=512+32=\mathbf{5 4 4}$, compared to $2^{9}+2^{4}+2^{7}=512+16+128=\mathbf{6 5 6}$ of $\boldsymbol{\Lambda}_{\mathbf{5}}$. As mentioned in the previous section, the same reduction in coding gain will be experienced in the first two decoding stages of both $\mathbf{\Lambda}_{\mathbf{4}}$ and $\boldsymbol{\Lambda}_{\mathbf{5}}$, while the use of a UEP code in the third stage of $\boldsymbol{\Lambda}_{\mathbf{5}}$ provides UEP capabilities. The proposed code achieves increased error performance and UEP capabilities at the expense of a $20 \%$ increase in decoding complexity, using multistage decoding, with respect to the multilevel code using conventional linear block codes.

\section{CONCLUSIONS}

It has been shown that the use of a binary LUEP code as component code in the multilevel construction produces enhanced UEP capabilities and increased error performance, at a modest reduction in bandwidth efficiency and a relatively small increase in decoding complexity. The proposed constructions offer a good trade-off between bandwidth efficiency, error performance and decoding complexity, which would otherwise be impossible to achieve using conventional linear block codes. Although in this paper the use of a binary LUEP as a component code in the multilevel construction is considered, it is possible to use two binary LUEP codes as component codes in the second and third encoding stages, if bandwidth efficiency and decoding complexity constraints allow it. 
Finally, it should be noted that in this research work a uniform 8-PSK signal set is used, as opposed to previously proposed BCM [5] which use nonuniform 8-PSK signal constellations. The improvement in error performance for part of the information symbols (UEP) is achieved here by increasing the Hamming distance between codewords in one or more of the encoding levels, through the use of LUEP codes, as opposed to increasing the product distance directly in the signal space as is the case in a nonuniform signal set. One problem with the use of nonuniform signal sets is that the increase in error performance for the most important information bits is usually obtained at the expense of a (sometimes considerable) performance degradation for other information parts. In this paper, multilevel constructions of block coded 8-PSK modulation for fading channels have been presented that achieve excellent error performance with no degradation of any information part.

Future research activities include the generalization of the examples presented in this paper to families of block coded 8-PSK modulations with unequal error protection capabilities.

\section{ACKNOWLEDGEMENTS}

This work was supported in part by NASA under Grant NAG 5-931, by the NSF under Grants NCR88813480 and NCR-9115400, and by the Japanese Society for the Promotion of Science (JSPS) under Postdoctoral Fellowship ID No. 93157.

\section{REFERENCES}

[1] H. Imai and S. Hirakawa, "A New Multilevel Coding Method Using Error Correcting Codes," IEEE Trans. Info. Theory, vol. IT-23, no. 3, pp. 371-376, May 1977.

[2] B. Masnick and J. Wolf, "On Linear Unequal Error Protection Codes," IEEE Trans. Info. Theory, vol. IT13, no. 4, pp. 600-607, Oct. 1967.

[3] J. Hagenauer, N. Seshadri and C.-E. W. Sundberg, "The Performance of Rate-Compatible Punctured Convolutional Codes for Digital Mobile Radio," IEEE Trans. Communications, vol. 38, no. 7, pp. 966980, July 1990.

[4] R.H. Morelos-Zaragoza and S. Lin, "Block QPSK Modulation Codes With Two Levels of Error Protection," Proceedings of the Fifth IEEE International Symposium on Personal, Indoor and Mobile Communications (PIMRC'94), vol. II, pp. 548-552, The Hague, The Netherlands, Sept. 1994.
[5] N. Seshadri and C.-E. W. Sundberg, "Coded Modulation with Time Diversity, Unequal Error Protection, and Low Delay for the Rayleigh Fading Channel," 1st. Conference on Universal Personal Communications (ICUPC '92), Conf. Rec., pp. 283-287, Dallas, Texas, Sept. 1992.

[6] N. Seshadri and C.-E. W. Sundberg, "Multilevel Trellis Coded Modulations for the Rayleigh Fading Channel," IEEE Trans. Communications, vol. 41, no. 9, pp. 13001310, Sept. 1993.

[7] J. Wu and S. Lin, "Multilevel Trellis MPSK Modulation Codes for the Rayleigh Fading Channel," IEEE Trans. Communications, vol. 41, no. 9, Sept. 1993.

[8] I.M. Boyarinov and G.L. Katsman, "Linear Unequal Error Protection Codes," IEEE Trans. Info. Theory, vol. IT-27, no. 2, pp. 168-175, March 1981.

[9] S. Rajpal, D. Rhee and S. Lin, "Low-Complexity and High-Performance Multilevel Coded Modulation for the AWGN and Rayleigh Fading Channels," to appear in IEEE Trans. Communications, vol. 43, no. 3, March 1995.

[10] W.J. Van Gils, "Two Topics on Linear Unequal Error Protection Codes: Bounds on Their Length and Cyclic Code Classes," IEEE Trans. Info. Theory, vol. IT-29, no. 6, pp. 866-876, Nov. 1983.

[11] A.E. Brouwer and T. Verhoeff, "An Updated Table of Minimum-Distance Bounds for Binary Linear Codes," IEEE Trans. Info. Theory, vol. 39, no. 2, pp. 662-677, Mar. 1993. 